\title{
Maturation of functional type III secretion machinery by activation of anaerobic respiration in enterohaemorrhagic Escherichia coli
}

\author{
Hiroki Ando, Hiroyuki Abe, Nakaba Sugimoto and Toru Tobe \\ Division of Applied Bacteriology, Graduate School of Medicine, Osaka University, 2-2 \\ Yamadaoka, Suita, Osaka 565-0871, Japan
}

Correspondence

Toru Tobe

torutobe@bact.med.osaka-u.ac.jp

Received 4 August 2006

Revised 3 October 2006

Accepted 9 October 2006

\begin{abstract}
Enterohaemorrhagic Escherichia coli (EHEC) is a gastrointestinal pathogen that causes diarrhoea and more severe diseases in humans. A key feature of EHEC is the type III secretion system (TTSS), which translocates virulence factors (effectors) directly into host cells. In this study, the expression and secretion of effectors in EHEC grown under anaerobic conditions were examined. The secretion of effectors was greatly enhanced, without an increase in their expression levels, when EHEC was grown in the presence of specific electron acceptors, such as trimethylamine $N$-oxide (TMAO) and nitrate, for anaerobic respiration. The activation of the TTSS was dependent on the activity of respiratory systems, including electron-acceptor-specific signalling systems and reductases. Although de novo protein synthesis was not required for TTSS activation, the inhibition of respiratory activity abolished secretion. EHEC grown with either TMAO or nitrate possessed a more intact type III secretion (TTS) apparatus, including the needle protein EscF and the translocator protein EspA, than EHEC grown without an electron acceptor. These observations suggest that activation of either the TMAO- or the nitrate-specific respiratory system accelerates the maturation of functional TTS apparatus under anaerobic growth conditions.
\end{abstract}

\section{INTRODUCTION}

Enterohaemorrhagic Escherichia coli (EHEC) is a lifethreatening human pathogen that causes haemorrhagic colitis, bloody diarrhoea, and haemolytic uraemic syndrome (Nataro \& Kaper, 1998). EHEC is a member of the attaching and effacing (A/E) pathogens (Nataro \& Kaper, 1998; Tzipori et al., 1995), which is a group that includes enteropathogenic E. coli (EPEC) (Moon et al., 1983) and the mouse pathogen Citrobacter rodentium (Schauer \& Falkow, 1993). $\mathrm{A} / \mathrm{E}$ lesions are characterized by the localized destruction of brush border microvilli, intimate attachment of the organism to the host cell membrane, and the formation of an actin-rich underlying structure in the host cell (Frankel et al., 1998). The genes essential for causing the $\mathrm{A} / \mathrm{E}$ lesions are encoded in a pathogenicity island designated the locus of enterocyte effacement (LEE). The LEE consists of more than 40 genes that encode the components of the type III secretion system (TTSS), including secreted proteins, chaperones, and adhesin and transcription regulators (Garmendia et al., 2005).

Abbreviations: $A / E$, attaching and effacing; DIC, differential interference contrast; EHEC, enterohaemorrhagic Escherichia coli; EPEC, enteropathogenic E. coli; LEE, locus of enterocyte effacement; TMAO, trimethylamine $\mathrm{N}$-oxide; TTS, type III secretion; TTSS, type III secretion system.
The TTSS is associated with many Gram-negative pathogens that cause disease in humans, animals, insects and plants (Galan \& Collmer, 1999). Features of the TTSS include: (i) the formation of a macromolecular complex that spans both membranes, and extends a needle-like projection out of the cell; and (ii) the delivery of virulence factors directly into the host cell (O'Connell et al., 2004). Once injected into the host cell, the virulence factors, referred to as effectors, co-opt the signalling pathways of the cell to promote responses beneficial to the pathogen (Galan \& Collmer, 1999). The TTSS of EPEC and EHEC is unique in that it attaches a filamentous extension to the needle complex (Sekiya et al., 2001; Wilson et al., 2001). The filament is composed of EspA, and appears to function as the conduit for the translocation of effector proteins. The effectors are delivered into the host cell through a translocation pore formed in the plasma membrane of the host cell by the translocator proteins EspB and EspD (Hartland et al., 2000; Ide et al., 2001).

Expression and secretion of the virulence factors are tightly regulated, and respond to environmental stimuli, such as temperature, $\mathrm{pH}$ and nutrient availability (Beltrametti et al., 1999; Kenny et al., 1997). Such regulation permits the coordinated and timely expression of the proteins required for virulence expression under changing environmental conditions. Clarification of the environmental factors and signal transduction systems that control virulence 
expression is important to improve our understanding of pathogenesis, and to identify drug targets. Most of the studies on the expression of virulence genes in EHEC have been carried out with bacteria grown under aerobic conditions, although the intestinal tract, which is the target site of EHEC infection, is anaerobic. Like other E. coli strains, EHEC is a facultative anaerobe, and it possesses a large number of systems for regulating anaerobic respiration in response to whatever terminal electron acceptor is available. These regulatory systems channel electrons from the donor to terminal acceptors, such that the overall difference in potential is maximized for any given growth condition (Shalel-Levanon et al., 2005). The terminal electron acceptors used in anaerobic respiration are usually low-molecular-weight oxygen-containing molecules, such as trimethylamine $\mathrm{N}$-oxide (TMAO), DMSO, nitrate and fumarate, which are abundant in the environment.

In this study, we performed what we believe to be the first investigation of the expression and type III secretion (TTS) of virulence factors occurring in response to electron acceptors under anaerobic conditions. Our results suggest that specific electron acceptors, TMAO and nitrate, enhance TTS by accelerating the formation of the fully assembled functional TTS apparatus.

\section{METHODS}

Bacterial strains, plasmids and growth conditions. The bacterial strains and plasmids used in this study are listed in Table 1. Unless otherwise specified, the bacteria were pre-cultured in $2 \mathrm{ml}$ Luria-Bertani (LB) medium for $14 \mathrm{~h}$ at $30^{\circ} \mathrm{C}$, with shaking, and then $100 \mu \mathrm{l}$ of the culture was used to inoculate $10 \mathrm{ml}$ fresh LB medium, both with and without an electron acceptor $(10 \mathrm{mM})$, in a $15 \mathrm{ml}$ tube. The bacteria were further cultured for $3 \mathrm{~h}$ at $37^{\circ} \mathrm{C}$ in an anaerobic jar, and for $3 \mathrm{~h}$ at $37^{\circ} \mathrm{C}$ without shaking (standing conditions). To inhibit protein synthesis and respiration, excess amounts of chloramphenicol $\left(100 \mu \mathrm{g} \mathrm{ml}^{-1}\right)$ or amytal $(5 \mathrm{mM})$ were added to the bacterial culture grown without an electron acceptor. After $30 \mathrm{~min}$ incubation at $37^{\circ} \mathrm{C}$, an electron acceptor was added, and the bacteria were incubated for an additional $1 \mathrm{~h}$ at $37^{\circ} \mathrm{C}$. The electron acceptors used were TMAO (Sigma), DMSO (Wako), nitrate (Sigma), and fumarate (Wako).

Construction of deletion mutants. To construct deletion mutants, we employed a one-step inactivation method (Datsenko \& Wanner, 2000). Briefly, PCR products containing the chloramphenicol-resistance cassette flanked by $40 \mathrm{bp}$ of sequence corresponding to the $5^{\prime}$ and $3^{\prime}$ ends of each gene were introduced by electroporation into the EHEC Sakai strain harbouring pKD46, which is a helper plasmid encoding the $\lambda$-Red, Gam and Bet proteins (Datsenko \& Wanner, 2000). The chloramphenicol-resistant colonies were examined for the correct chromosomal structures by PCR tests using locus-specific primers. The DNA sequences of the primers are indicated in Table 2.

Adherence assay. Bacterial cultures grown as described above were added directly to tissue culture wells that contained a confluent monolayer of Caco-2 cells growing on glass coverslips $(13 \mathrm{~mm}$ diameter). After $2 \mathrm{~h}$ incubation at $37^{\circ} \mathrm{C}$, the cells were washed five times with PBS to remove non-adherent bacteria. The cells were then incubated in fresh medium for an additional $1.5 \mathrm{~h}$ at $37^{\circ} \mathrm{C}$. After washing with PBS, the cells were fixed with $100 \%$ methanol for $10 \mathrm{~min}$ at room temperature, and stained with Giemsa staining solution. The adherence efficiency was determined by counting the number of bacteria adhering to the Caco- 2 cells.

Electrophoresis and immunoblot analysis. SDS-PAGE $(8-13.5 \%)$ or Tricine-SDS-PAGE $(16 \%)$ was used throughout this

Table 1. Bacterial strains and plasmids used in this study

\begin{tabular}{|c|c|c|}
\hline Strain or plasmid & Description & Source or reference \\
\hline \multicolumn{3}{|l|}{ Strains } \\
\hline Sakai (RIMD 0509952) & WT EHEC O157:H7 & Hayashi et al. (2001) \\
\hline SKI0073 & Sakai $\Delta($ torSTR $)::$ cat & This study \\
\hline SKI0156 & Sakai $\Delta$ narQ: : cat & This study \\
\hline SKI0171 & Sakai $\Delta($ narXL $)::$ cat & This study \\
\hline SKI0194 & Sakai $\Delta$ narP:: cat & This study \\
\hline SKI1188 & Sakai $\Delta($ narVWYZ) ::cat & This study \\
\hline SKI1189 & Sakai $\Delta($ narGHJI) $::$ cat & This study \\
\hline SKI1191 & Sakai $\triangle($ torCAD $):$ : cat & This study \\
\hline SKI1193 & Sakai $\Delta p u r K:$ : cat & This study \\
\hline SKI1195 & Sakai $\Delta e s c D:$ : cat & This study \\
\hline SKI1197 & Sakai $\Delta u b i E:$ : cat & This study \\
\hline SKI1201 & Sakai $\Delta m e n A:$ : cat & This study \\
\hline \multicolumn{3}{|l|}{ Plasmids } \\
\hline pKD3 & $\begin{array}{l}\text { Template plasmid for } \lambda \text {-Red } \\
\text { recombination system, } \text { Ap }^{\mathrm{r}}\end{array}$ & $\begin{array}{l}\text { Datsenko \& Wanner } \\
\text { (2000) }\end{array}$ \\
\hline pKD46 & $\begin{array}{l}\text { Red recombinase expression } \\
\text { plasmid, } \mathrm{Ap}^{\mathrm{r}}\end{array}$ & $\begin{array}{l}\text { Datsenko \& Wanner } \\
(2000)\end{array}$ \\
\hline pGEM-T-easy & TA cloning vector, $A^{r}{ }^{r}$ & Promega \\
\hline pGEM-grlA & pGEM-T-easy-grlA & This study \\
\hline
\end{tabular}


Table 2. Oligonucleotide primers used in this study

\begin{tabular}{|c|c|}
\hline Primer & Sequence $\left(5^{\prime} \rightarrow 3^{\prime}\right)$ \\
\hline grlA-U & GAATATGGAAAATGGAATCT \\
\hline grlA-D & CCGAGAAAAAGGCTTACCCTGG \\
\hline torSTR-H1 & GAATATGAACAGATATGAACAGAATGAGTAAAACCCTCTGGTGTAGGCTGGAGCTGCTTC \\
\hline torSTR-H3 & ATGCCACTATTCTAGTTATTCTTAACTGGCTGATATTGATATATGAATATCCTCCTTAGT \\
\hline torCAD-H1 & TCATTAAGATCGCTTCACTAAACCATAATTTTACAGGGGCGTGTAGGCTGGAGCTGCTTC \\
\hline torCAD-H2 & ATGCGACGCTAAACGCGTCTTATCAGGCTAACAAACGACGATATGAATATCCTCCTTAGT \\
\hline NarP-H1 & CTCAAACATAATGATTCTAATAAAACCTCAGGAGACTACTGTGTAGGCTGGAGCTGCTTC \\
\hline NarP-H2 & AAATAAAAAGAGGCCCGATGGTGATGCCATCGGGCTATTTATATGAATATCCTCCTTAGT \\
\hline NarQ-H1 & CGAACTGGAACATTAATGATTTTTTGTGGAGAAGACGCGTGTGTAGGCTGGAGCTGCTTC \\
\hline NarQ-H2 & ACCTTAAGTGCAAGTATTCTTTGGTCAGTAGGAGGCATTAATATGAATATCCTCCTTAGT \\
\hline NarXL-H1 & AAGGTTATTGCTCATTTAAAGCCTGAAGGAAGAGGTTTACGTGTAGGCTGGAGCTGCTTC \\
\hline NarXL-H2 & TGATGCACCCATACCGCTGCTTCCACGCGAGACTTGAGCTATATGAATATCCTCCTTAGT \\
\hline narVWYZ-H5 & CGTAGTCTAGGGTTAAGTAAGGACCTCGTCCTCAGTACAGGTGTAGGCTGGAGCTGCTTC \\
\hline narVWYZ-H6 & CCTCTTGATAGTATCGGACGTTCACCGGCCTCTCGCTTCCCATATGAATATCCTCCTTA \\
\hline narGHJI-H1 & ATATGTTACCCATGGGGAATACTCCTTAATACCCATTTGCGTGTAGGCTGGAGCTGCTTC \\
\hline narGHJI-H2 & TGTAGGTCGGATAAGCGAAGCGCATCCGACAACTCACCCCATATGAATATCCTCCTTAGT \\
\hline escD-H1 & TTTCTCATTGTTCAACCACCAGGATGAATAAAATTTAAAAGTGTAGGCTGGAGCTGCTTC \\
\hline escD-H2 & CAATAAAAAACCCTCCGAAGAGGGGGAAGAGGATAAGAAAATATGAATATCCTCCTTAGT \\
\hline ubiE-H1 & GGGCATCGACGCCCAATCTGTTACACTTCTGGAACAATTTGTGTAGGCTGGAGCTGCTTC \\
\hline ubiE-H2 & CTGCCGTCACTAAAGGTTTAAAAGGCATTTCCGGTCTCCTCATATGAATATCCTCCTTA \\
\hline menA-H1 & CAAACTACCCCGACTTTCCGGGGTAAAAATAACCGCGCATGTGTAGGCTGGAGCTGCTTC \\
\hline menA-H2 & CAGACAACCGTTTTAGTAGTTAACAATTAACTATAAACAGCATATGAATATCCTCCTTA \\
\hline purK-H1 & CGGACCCGCGAGGTGCGGCATGAAACAGGTTTGCGTCCTCGTGTAGGCTGGAGCTGCTTC \\
\hline purK-H2 & CGGATAAGGCGTTCACGCCGCATCCGGCAATCAAATGAGCATATGAATATCCTCCTTA \\
\hline
\end{tabular}

study (Laemmli, 1970; Schagger \& von Jagow, 1987). For immunoblot analysis, proteins separated by SDS-PAGE or Tricine-SDSPAGE were transferred to an Immobilon-P transfer membrane (Millipore) or an Immuno-Blot PVDF membrane (Bio-Rad). The proteins on the membranes were detected using primary antibodies specific for EspA, EspB, Tir (Tatsuno et al., 2000), EscF (rabbit antibody raised against the MBP-EscF fusion protein), EscC (Sekiya et al., 2001), DnaK (mAb; Calbiochem) and FLAG (mAb; Sigma). Proteins were visualized with a horseradish-peroxidase-conjugated secondary antibody using an ECL detection kit (Amersham Biosciences).

\begin{abstract}
Analysis of proteins in culture supernatant and whole-cell extract. Bacteria grown as described above were harvested from $1 \mathrm{ml}$ culture by centrifugation. The bacterial pellet was dissolved in SDS-sample buffer $\left(100 \mu \mathrm{l}\right.$ per $\mathrm{OD}_{600}$ unit of original culture). To prepare the proteins from the culture supernatant, $10 \mathrm{ml}$ culture was spun, and the supernatant was passed through a filter $(0.22 \mu \mathrm{m}$ pore size; Millipore). Proteins in the filtered supernatant were precipitated by $6 \%$ (final) trichloroacetic acid, and dissolved in SDS sample buffer. The proteins were separated by SDS-PAGE or Tricine-SDS-PAGE, and detected by immunoblotting or Coomassie brilliant blue staining.
\end{abstract}

Immunofluorescence. A drop of bacterial culture prepared as described above was placed on a glass coverslip (13 $\mathrm{mm}$ diameter), and the bacteria were fixed by air drying in $4 \%$ paraformaldehyde for $50 \mathrm{~min}$ at $37^{\circ} \mathrm{C}$. The coverslips were washed twice with PBS, and blocked in PBS containing $4 \%$ BSA for $30 \mathrm{~min}$ at room temperature. The washed bacteria were then incubated with a rabbit polyclonal anti-EspA antibody for $1 \mathrm{~h}$ at $37^{\circ} \mathrm{C}$. After two washes, the coverslips were incubated with Alexa-488-labelled goat antirabbit secondary antibody for $1 \mathrm{~h}$ at $37^{\circ} \mathrm{C}$ to stain EspA filaments.
The coverslips were washed twice, and mounted onto glass slides using $2 \mu \mathrm{l}$ Vectashield (Vector Laboratories). The fluorescence was observed under a confocal laser scanning microscope, and the bacteria were examined with Nomarski differential interference contrast (DIC; Leica).

Measurement of the relative ATP concentration. The relative ATP concentration of the bacterial whole-cell lysate was measured using an ATP determination kit (Molecular Probes), as described in the manufacturer's instructions, with slight modification. A $10 \mu \mathrm{l}$ volume of bacterial culture, prepared as described above, was mixed with $90 \mu \mathrm{l}$ standard reaction solution containing polymyxin B sulfate $\left(1 \times 10^{4}\right.$ units $\left.\mathrm{ml}^{-1}\right)$ in a luminometer cuvette. The reaction mixture was vortexed briefly, and the luminescence was measured immediately in a Lumat LB9501 luminometer (Berthold). The luminescence readings were carried out for $10 \mathrm{~s}$. The luminescence measurements were normalized to the bacterial density of each culture, which was measured as the $\mathrm{OD}_{600}$ value.

Isolation of the TTS apparatus. The TTS apparatus was isolated by adapting a method described by Kubori et al. (1998). Overnight cultures $(8 \mathrm{ml})$ of bacteria harbouring multiple copies of grlA grown in $\mathrm{LB}$ at $30^{\circ} \mathrm{C}$, with shaking, were diluted in $800 \mathrm{ml} \mathrm{LB}$, both with and without an electron acceptor (TMAO and nitrate), and the bacteria were then grown without shaking for $4 \mathrm{~h}$ at $37^{\circ} \mathrm{C}$. The bacteria were collected by centrifugation, and gently suspended in $80 \mathrm{ml}$ icecold sucrose solution ( $500 \mathrm{mM}$ sucrose, $150 \mathrm{mM}$ Tris, $\mathrm{pH}$ 8.0). The suspension was mixed with $4 \mathrm{ml} 10 \mathrm{mg}$ lysozyme $\mathrm{ml}^{-1}$, and then with $320 \mu \mathrm{l} 500 \mathrm{mM} \mathrm{Na}{ }_{2}$ EDTA, and stirred on ice for $1 \mathrm{~h}$. After incubation at $37^{\circ} \mathrm{C}$ for $15 \mathrm{~min}$ with stirring, the suspension was mixed with $8 \mathrm{ml} 3 \%$ Triton X-100, and incubated at room temperature until the solution became clear. After adding $400 \mu \mathrm{l} 1 \mathrm{M}$ $\mathrm{MgSO}_{4}$ and $5.28 \mathrm{ml} 5 \mathrm{M} \mathrm{NaCl}$, the sample was spun at $20000 \mathrm{~g}$ for 
20 min at $4{ }^{\circ} \mathrm{C}$ to remove unlysed cells and cell debris, and then the supernatant was spun at $100000 \mathrm{~g}$ for $1 \mathrm{~h}$ at $4{ }^{\circ} \mathrm{C}$. The pellet was suspended in TET buffer (10 mM Tris, pH 8.0, $2 \mathrm{mM}$ EDTA and $0.1 \%$ Triton $\mathrm{X}-100$ ), and subjected to density-gradient centrifugation with $12 \mathrm{ml} 36 \% \mathrm{CsCl}$ for $17 \mathrm{~h}$ at $20^{\circ} \mathrm{C}$. Macromolecules in $10 \mathrm{ml}$ of the middle portion of the gradient were spun down at $100000 \mathrm{~g}$ for $1 \mathrm{~h}$ at $4{ }^{\circ} \mathrm{C}$. After washing with TET buffer, the pellet was dissolved in sample buffer or TET buffer for immunoblotting.

\section{RESULTS}

\section{Effect of electron acceptors on TTS in EHEC grown under anaerobic conditions}

We compared the growth and expression of virulence factors in EHEC grown under two low $\mathrm{O}_{2}$ conditions, anaerobic and standing, with those in EHEC grown under aerobic conditions. Under both of the low $\mathrm{O}_{2}$ conditions, the growth rate of EHEC in the exponential phase was slower, and its cell density in the stationary phase was lower, than when it was grown under aerobic conditions. The expression level of the LEE-encoded virulence factors EspB and Tir was comparable between EHEC grown under low $\mathrm{O}_{2}$ and aerobic conditions. However, the amounts of EspB and Tir in the culture supernatant of EHEC grown under the low $\mathrm{O}_{2}$ conditions were much lower than in the supernatant of the aerobic culture (Fig. 1a). Since the growth of EHEC under the low $\mathrm{O}_{2}$ conditions was slower than under aerobic conditions, we added terminal electron acceptors to the medium, as an alternative to $\mathrm{O}_{2}$, and stimulated EHEC growth. All four electron acceptors tested, i.e. TMAO, DMSO, nitrate and fumarate, affected the growth in a similar manner: the growth rate in the exponential phase was not changed, whereas the growth after the transition from the exponential to the stationary phase was stimulated (Fig. 1b). We next examined the expression and secretion of virulence factors in EHEC grown in early stationary phase in the presence of an electron acceptor. Irrespective of the growth medium, the amount of EspB or Tir protein expressed in bacteria grown with an electron acceptor was almost the same as for bacteria grown without an electron acceptor. However, the amount of EspB and Tir in the culture supernatant of bacteria grown with an electron acceptor was greater than in the supernatant of bacteria grown without an electron acceptor (Fig. 1c). Although the effect on growth was not very different among the electron acceptors we tested, the effect of TMAO and nitrate on TTS was much stronger than that of the other electron acceptors. These results suggest that the increase in secretion is not the result of growth stimulation, but a result of the action of other mechanisms that specifically respond to TMAO and nitrate. The increase in EspB and Tir in the culture supernatant was not the result of cell lysis or

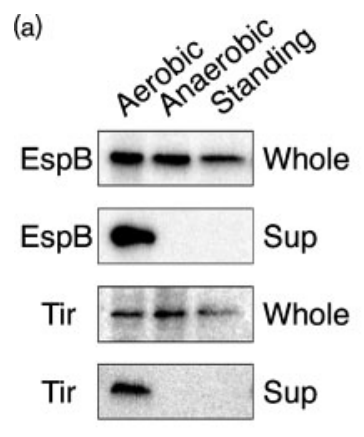

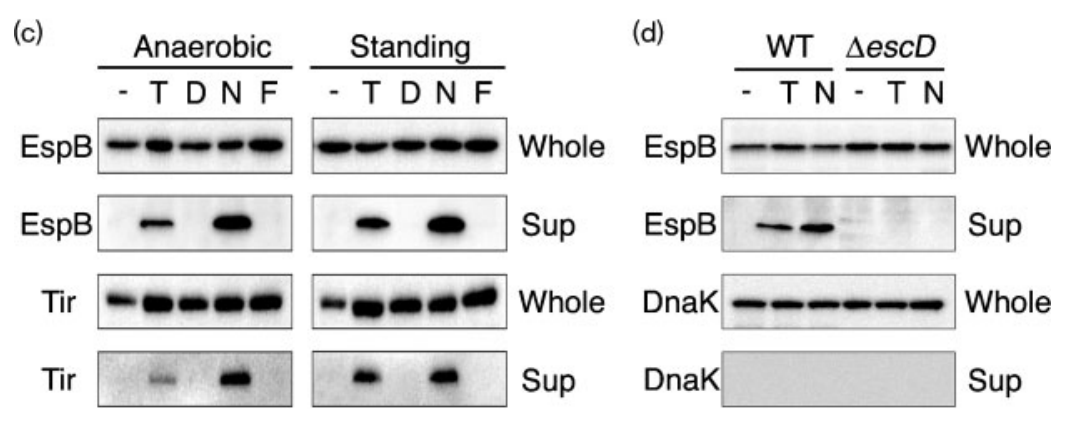
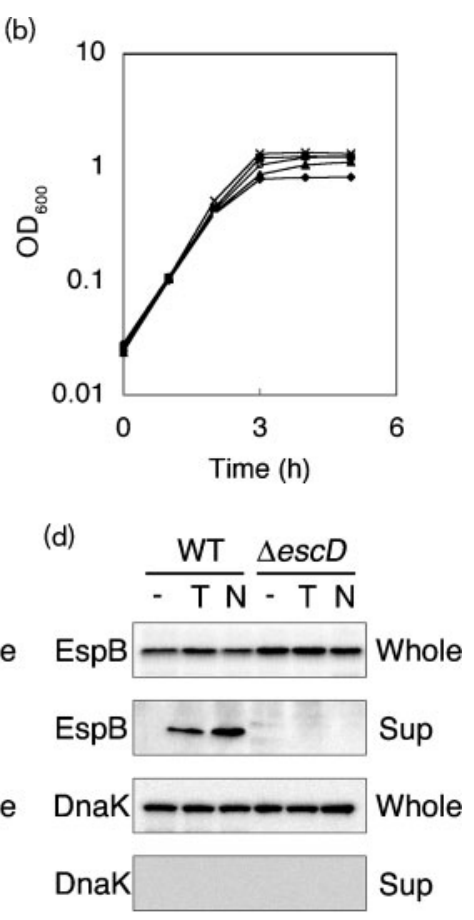

Fig. 1. Expression and secretion of type III secreted protein in EHEC grown under anaerobic conditions. (a) Expression and secretion of EspB and Tir in EHEC grown under aerobic, anaerobic and standing conditions. Bacteria were grown in LB with vigorous shaking (aerobic), in an anaerobic jar (anaerobic), and without shaking (standing), at $37^{\circ} \mathrm{C}$ for $3 \mathrm{~h}$. EspB and Tir in whole-cell extracts and culture supernatant were detected by immunoblotting with anti-EspB and anti-Tir antibodies, respectively. (b) Growth of EHEC in LB, both with and without an electron acceptor, at $37^{\circ} \mathrm{C}$. Bacterial cell densities were monitored by $\mathrm{OD}_{600}$ readings. No electron acceptor; $\mathbf{\square}$, TMAO; $\boldsymbol{\Delta}$, DMSO; $\times$, nitrate; $\bigcirc$, fumarate. (c) Effect of electron acceptors on the expression and secretion of EspB and Tir. Bacteria were grown in LB under anaerobic and standing conditions at $37^{\circ} \mathrm{C}$ for $3 \mathrm{~h}$, both with and without an electron acceptor. The proteins in the culture supernatant and whole-cell extract were analysed as described for (a). (d) Effect of electron acceptors in an EHEC TTSS-deficient mutant. The proteins in the culture supernatant and wholecell extract were analysed as described for (a). $\Delta e s c D$, mutant lacking a functional TTSS; Whole, whole-cell extract; Sup, culture supernatant; T, TMAO; D, DMSO; N, nitrate; $F$, fumarate; -, no addition. 
TTSS-independent secretion, because the cytoplasmic protein DnaK was not detected in the supernatant, and neither EspB nor Tir was present in the culture supernatant of the $e s c D$ mutant of EHEC, which lacked a functional TTSS (Fig. 1d).

The increase of TTS in response to either TMAO or nitrate is a general phenotype among EHEC O157 strains, since the increase in TTS was observed in all of the eight strains tested (data not shown), which are representative of EHEC O157 subgroups (Ogura et al., 2006; Ohnishi et al., 2002).

\section{Activation of anaerobic respiratory systems is necessary for TMAO- and nitrate-enhanced TTS}

To explore the role of anaerobic respiratory systems in the enhancement of TTS, we examined the effect of a respiratory inhibitor on this process. Bacteria were grown in LB without an electron acceptor for $3 \mathrm{~h}$, and then a respiration inhibitor, amytal, was added $30 \mathrm{~min}$ prior to the addition of the electron acceptor. Even though the EHEC was grown to the transition phase without an electron acceptor, further incubation with either TMAO or nitrate stimulated the secretion of EspB. In contrast, prior treatment with amytal completely abolished the stimulation of EspB secretion by TMAO and nitrate (Fig. 2a). Moreover, we examined the
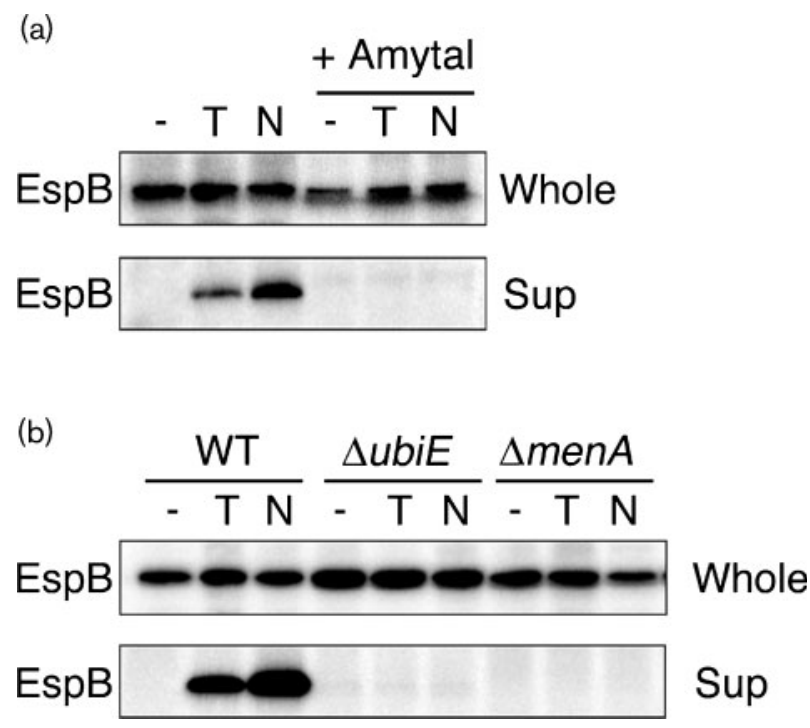

Fig. 2. Roles of respiratory systems in the activation of TTS by electron acceptors. (a) Effect of a respiratory inhibitor on the activation of TTS. Bacteria were grown in LB under standing conditions at $37^{\circ} \mathrm{C}$ for $3 \mathrm{~h}$, without an electron acceptor, and then exposed to amytal $(5 \mathrm{mM})$ for $30 \mathrm{~min}$ at $37^{\circ} \mathrm{C}$ prior to incubation with an electron acceptor for $1 \mathrm{~h}$. EspB protein in the culture supernatant and whole-cell extract was detected by immunoblotting using specific antibody. (b) Enhancement of TTS in mutants deficient in quinone synthesis. Bacteria were grown in LB under standing conditions at $37^{\circ} \mathrm{C}$, both with and without an electron acceptor. Abbreviations, see legend to Fig. 1. effect of TMAO and nitrate on the TTS in a series of mutants that were deficient in quinone synthesis. Quinones are lipid-soluble molecules that are essential components in respiratory electron transfer chains. To explore the role of quinones in the enhancement of TTS, the TTS response of mutants defective in quinone synthesis was compared with that of the wild-type (WT) strain. In E. coli, the ubiE mutant is deficient in the synthesis of ubiquinone and menaquinone, whereas the menA mutation abolishes the synthesis of menaquinone and demethylmenaquinone (Lee et al., 1997; Stevenson et al., 1998; Wissenbach et al., 1992). In EHEC, both the $u b i E$ mutant and the menA mutant impaired the enhancement of EspB secretion in response to TMAO and nitrate (Fig. 2b). These results strongly suggest that activation of the respiratory system is necessary for activation of TTS in EHEC grown under anaerobic conditions.

\section{A respiration system specific for TMAO or nitrate is necessary for enhanced TTS}

At the final stage of respiration, electrons are transferred from quinones to the terminal electron acceptor by using an electron-acceptor-specific reductase. TMAO reductase, TorA, is encoded by the tor $C A D$ operon, and its expression is activated by the TorSTR signalling system (Ansaldi et al., 1999; Mejean et al., 1994; Pommier et al., 1998). E. coli also possesses three distinct nitrate reductases. The narGHJI operon encodes the major respiratory nitrate reductase located in the cytoplasmic membrane, and its expression is activated by the NarXL signalling system (Darwin et al., 1996; Walker \& DeMoss, 1994). The narVWYZ operon encodes a second nitrate reductase, which is biochemically similar to the NarGHJI enzyme, but is constitutively produced at relatively low levels in the cell (Blasco et al., 1990). A third nitrate reductase, encoded by the napFDAGHBC operon, is located in the periplasm, and its production is activated by the NarPQ signalling system, but not by NarXL (Darwin \& Stewart, 1995; Darwin et al., 1998; Stewart, 2003). To explore the role of electron acceptors in activation of TTS, we first examined a series of mutants that were deficient in either the TMAO- or the nitrate-specific signalling system, as this is necessary for activation of the corresponding expression of the gene encoding the reductase. The TTS response to TMAO or nitrate was abolished by the defect in the signaling system, i.e. the torSTR and narXL deletion mutations, respectively, without affecting the level of EspB expression in the cell (Fig. 3a). On the other hand, the response of TTS to nitrate was unaffected by deletion of the NarP-NarQ system. Deletion of the nitrate-specific signalling systems NarX-NarL and NarP-NarQ did not affect the response to TMAO, and vice versa (Fig. 3a). Next, we examined the response in a series of mutants that were deficient in either TMAO- or nitrate-specific reductase. The TTS response to TMAO was abolished completely by the torCAD mutation, but was not affected by the narGHJI mutation. In contrast, the nitrate-enhanced secretion was greatly decreased by the narGHJI mutation, but was not affected by the torCAD mutation (Fig. 3b). The NarVWYZ 

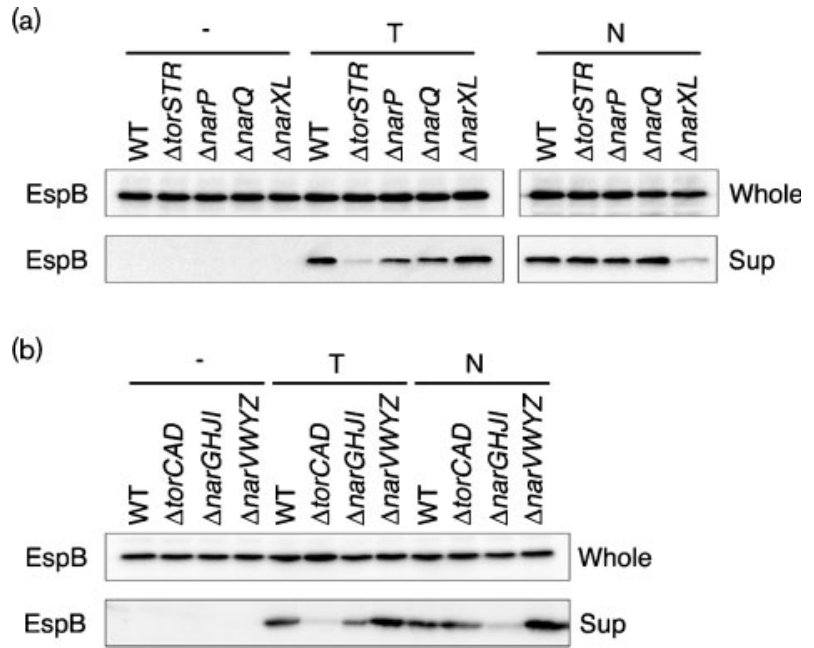

Fig. 3. Role of the TMAO- and nitrate-specific respiration systems in the activation of TTS by an electron acceptor. (a) Role of TMAO- and nitrate-specific signal transduction systems. Bacteria were grown in LB under standing conditions at $37^{\circ} \mathrm{C}$ for $3 \mathrm{~h}$, with and without an electron acceptor (T, TMAO; $\mathrm{N}$, nitrate). EspB in culture supernatant (Sup) and whole-cell extract (Whole) was detected by immunoblotting. $\Delta$ torSTR, mutant lacking the TMAO-specific signalling system; $\triangle$ narQ, mutant lacking the nitrate-specific sensor kinase; $\triangle$ nar $P$, mutant lacking the nitrate-specific response regulator; $\triangle$ nar $X L$, mutant lacking the nitrate-specific signalling system. (b) Role of the electron acceptor-specific reductase. $\triangle$ torCAD, mutant lacking TMAO reductase; $\triangle$ narGHJl, mutant lacking NarGHJl nitrate reductase; $\triangle$ narVWYZ, mutant lacking NarVWYZ nitrate reductase.

enzyme, which is constitutively produced at low levels, had no effect on the nitrate-enhanced TTS (Fig. 3b). These results clearly indicate that the activation of a respiratory system specific for either TMAO or nitrate is essential for the enhancement of TTS.

\section{Increase in ATP concentration by electron acceptors is not correlated with the level of TTS}

The TTSS needs ATP as a driving force to secrete virulence factors (Eichelberg et al., 1994; Hueck, 1998; Woestyn et al., 1994). Since the activation of respiration could increase the yield of ATP, one explanation for the enhancement of TTS by activating the respiratory system is that the increased ATP concentration stimulates TTS. To examine the correlation between ATP and the level of TTS, we measured the ATP concentration in bacteria grown both with and without an electron acceptor. Although the ATP concentration was correlated with the growth rate in the late exponential to early stationary phase, no correlation with the amount of secreted protein was observed (Figs 4 and 1b). All four electron acceptors used in this study enhanced ATP production, while DMSO and fumarate enhanced TTS at a much lower level than TMAO and nitrate. We also increased the ATP concentration in EHEC by using the purK mutant, and growing the bacteria in medium containing adenine. Although the concentration of ATP in the mutant grown with adenine was higher than in the WT grown with an electron acceptor (Fig. 4a), the secretion of EspB by the mutant remained very low, and was similar to that of the WT EHEC grown without an electron acceptor (Fig. 4b).

\section{Electron acceptors activate an existing TTS apparatus}

To determine whether the enhanced TTS required de novo protein synthesis, we examined the response after inhibiting protein synthesis. Bacteria were grown in LB without an electron acceptor for $3 \mathrm{~h}$, and then a translation inhibitor, chloramphenicol, was added for $30 \mathrm{~min}$ prior to the addition of the electron acceptor. Albeit reduced in the level of secretion, bacteria treated with chloramphenicol showed the TTS response to TMAO and nitrate (Fig. 5). Moreover, the inhibition of respiratory activity by amytal completely abolished the response to electron acceptors (Fig. 5). These

(a)

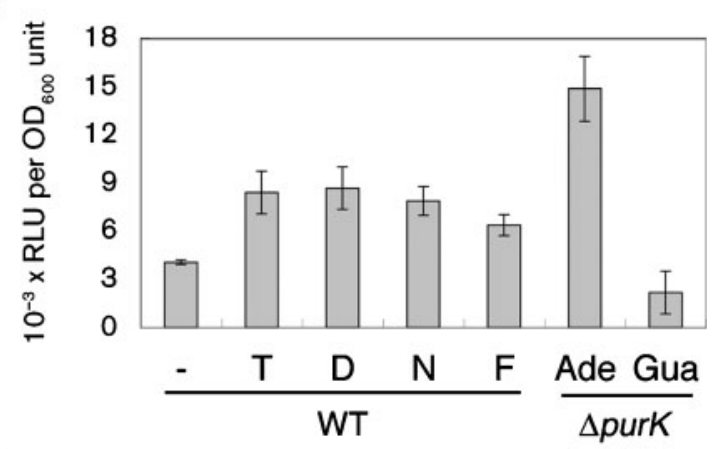

(b)

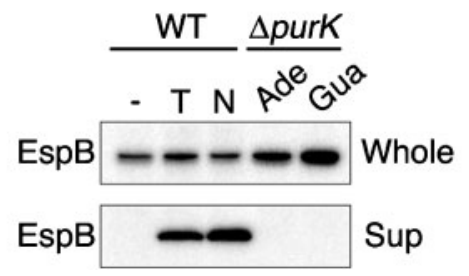

Fig. 4. Changes in the cellular concentration of ATP, and TTS activity. WT EHEC was grown in LB under standing condition at $37^{\circ} \mathrm{C}$ for $3 \mathrm{~h}$, both with and without an electron acceptor (T, TMAO; D, DMSO; N, nitrate; $\mathrm{F}$, fumarate). The purK mutant $(\triangle p u r K)$ was grown in Dulbecco's modified Eagle's medium (DMEM) under standing condition at $37^{\circ} \mathrm{C}$ for $3 \mathrm{~h}$, with adenine (Ade) and guanine (Gua). (a) Cellular ATP levels in WT EHEC grown both with and without an electron acceptor, and in the purK mutant grown with adenine and guanine. The relative ATP concentration of the bacterial lysate was measured using an ATP determination kit. Mean $( \pm S D)$ values were obtained from three independent experiments. (b) Expression and secretion of EspB in WT EHEC grown with an electron acceptor, and in the purK mutant grown in DMEM with adenine and guanine. Whole, whole-cell extract; Sup, culture supernatant. 
results support the idea that either TMAO or nitrate respiration is essential for the enhanced TTS described above, and they clearly indicate that the enhanced TTS could be achieved without de novo protein synthesis, including synthesis of the TTS apparatus. Thus, either TMAO or nitrate respiration activates an existing TTS apparatus.

\section{TMAO and nitrate facilitate maturation of the TTS apparatus}

The functional TTS apparatus of EHEC consists of a basal body with a needle structure attached to it by an EspAsheath-like structure. The EspA-sheath-like structure protrudes from the bacterial surface, and forms a filament that serves as a bridge, tethering the bacterium to the host cell (Knutton et al., 1998). To assess the effect of the electron acceptor on the formation of functional TTS apparatus, we observed the production of the EspA filament, and the adherence capacity of EHEC grown both with and without an electron acceptor. EspA filaments on the surface of EHEC were detected using immunofluorescence and an EspA-specific antibody (Fig. 6a). Compared with EHEC grown without an electron acceptor, EspA filaments on bacteria grown with TMAO and nitrate increased 5.4- and 3.5-fold, respectively. Because the adherence of EHEC to epithelial cells, and subsequent formation of microcolonies, depend mainly on TTSS activity, we next examined the adherence capacity of bacteria grown in LB with an electron acceptor (Fig. 6b). The adherence capacity of bacteria grown in LB with TMAO and nitrate increased 20.7- and 27.0-fold, respectively, over that of bacteria grown without an electron acceptor. The adherence capacity of the escD mutant was not increased by an electron acceptor, indicating that the increased adherence caused by TMAO and nitrate was dependent on the TTSS.

Since TTS was enhanced by the activation of a respiratory system without de novo protein synthesis, it seemed likely that the TTS apparatus changed from an inactive form to an

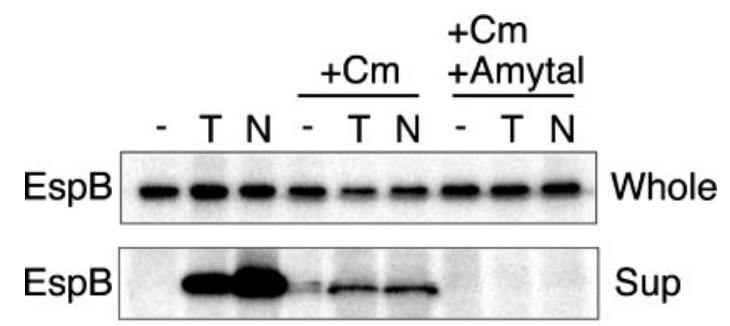

Fig. 5. Enhancement of TTS in EHEC, without translation activity. Bacteria were grown in LB under standing conditions at $37^{\circ} \mathrm{C}$ for $3 \mathrm{~h}$, without an electron acceptor, and then exposed to chloramphenicol $\left(\mathrm{Cm} ; 100 \mu \mathrm{g} \mathrm{ml}^{-1}\right)$, or chloramphenicol $\left(100 \mu \mathrm{g} \mathrm{ml}^{-1}\right)$ and amytal $(5 \mathrm{mM})$, for $30 \mathrm{~min}$ at $37^{\circ} \mathrm{C}$. The electron acceptor was added ( $\mathrm{T}, \mathrm{TMAO} ; \mathrm{N}$, nitrate), and the bacteria were incubated for a further $1 \mathrm{~h}$ at $37^{\circ} \mathrm{C}$. EspB in the whole-cell extract (Whole) or in the culture supernatant (Sup) was detected by immunoblotting.

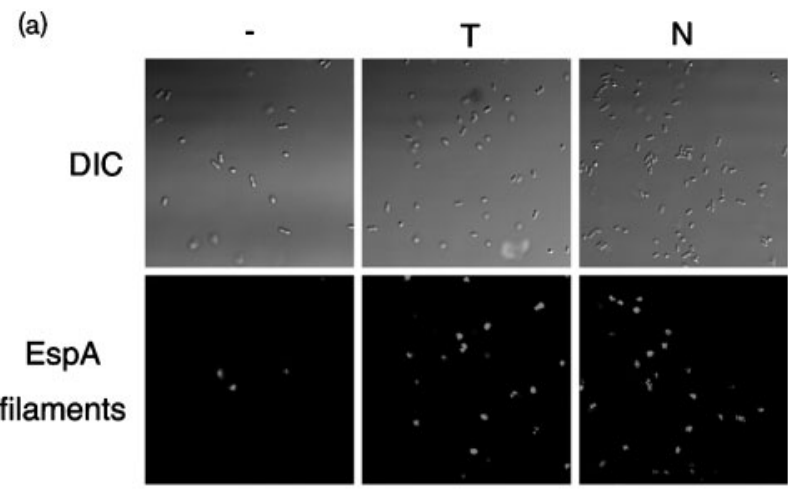

(b)

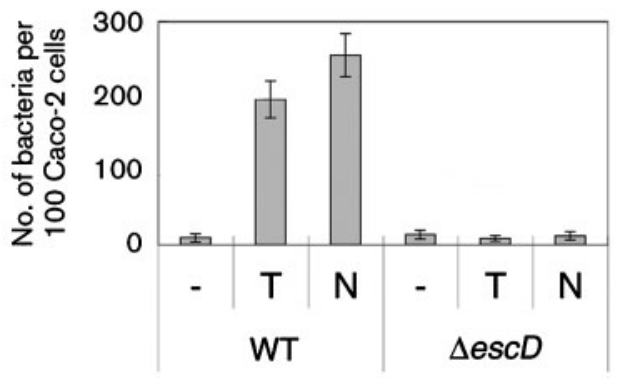

Fig. 6. Enhancement of EspA filament formation and adherence capacity in EHEC grown with TMAO and nitrate. (a) Production of EspA filament in EHEC grown under anaerobic conditions. Bacteria were grown in LB under standing conditions at $37^{\circ} \mathrm{C}$ for $3 \mathrm{~h}$, both with and without an electron acceptor (T, TMAO; $\mathrm{N}$, nitrate). Bacteria were fixed, and EspA was detected by immunofluorescent staining using anti-EspA antibody. The fluorescence was observed under a confocal laser scanning microscope. Bacteria were visualized by Nomarski DIC. (b) Adherence of bacteria to Caco-2 cells. Bacteria were grown in LB under standing condition at $37^{\circ} \mathrm{C}$ for $3 \mathrm{~h}$, both with and without an electron acceptor, and then allowed to infect Caco2 cells for $3.5 \mathrm{~h}$, which includes $1.5 \mathrm{~h}$ in fresh medium after the removal of non-infecting bacteria. Cells were fixed, and stained with Giemsa staining solution. The adherence efficiency was determined by counting the number of bacteria adhering to the Caco-2 cells. Means $( \pm S D)$ were obtained from three independent experiments. $\Delta \operatorname{esc} D$, mutant lacking functional TTSS.

active form in response to the activation of either TMAO or nitrate respiration. Recently, it was reported that there are two forms of TTS apparatus: the needle complex and the base complex in Salmonella typhimurium (Marlovits et al., 2004). In contrast to the fully assembled needle complex, the base complex, which is thought to be the preassembled complex, lacks the needle substructure. As suggested by the differences in the appearance of EspA filament on the bacterial surface (Fig. 6a), the TTS apparatus that formed in EHEC grown without an electron acceptor could be the base complex lacking the needle substructure. To investigate the presence of the needle component in the TTS apparatus, we partially purified the TTS apparatus by preparing fractions containing macromolecules from the bacterial membrane. 
We first tried to isolate the TTS apparatus in WT EHEC, but failed to detect any of the component proteins by using antibodies. We then increased the expression of the TTS apparatus by introducing multiple copies of the grlA gene, which encodes a positive transcriptional regulator for LEE genes (Deng et al., 2004). Although overexpression of the grlA gene increased the amount of translocators and effectors, growth with either TMAO or nitrate further stimulated the secretion markedly (Fig. 7a). The amount of EscC, the outer-ring protein of the basal body of the TTS apparatus (Gauthier et al., 2003), was also increased to a detectable level, and the amount of EscC was not altered by the presence of an electron acceptor (data not shown). The fraction containing the needle complex was prepared from EHEC overexpressing grlA, grown both with and without an electron acceptor, and it was analysed for the components of TTS apparatus, i.e. EscC and EscF. EscF is a needle structural protein (Sekiya et al., 2001). EscC was detected at the same level in bacteria irrespective of the growth conditions, while the amount of EscF protein was much greater in preparations from bacteria grown with either TMAO or nitrate, than from those grown without an electron acceptor (Fig. 7b). These results strongly suggest that, under anaerobic growth conditions, formation of the fully assembled needle complex is stimulated by the activation of specific (a)

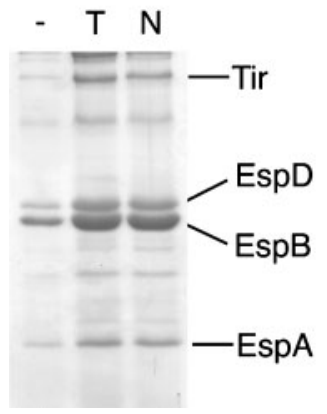

(b)

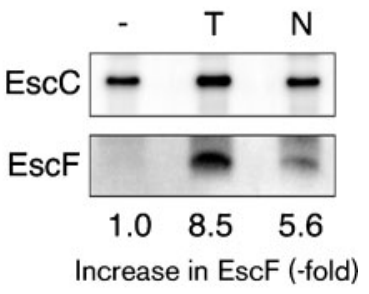

Fig. 7. Maturation of TTS apparatus. (a) Enhancement of TTS by an electron acceptor in EHEC overexpressing $g r / A$. Bacteria were grown in LB under standing conditions at $37^{\circ} \mathrm{C}$ for $4 \mathrm{~h}$, both with and without an electron acceptor (T, TMAO; N, nitrate). EspB in the culture supernatant was detected by Coomassie brilliant blue staining. (b) Amount of EscC and EscF in the TTS apparatus isolated from the bacterial membrane. EHEC overexpressing $g r l A$ was grown under standing conditions at $37^{\circ} \mathrm{C}$ for $4 \mathrm{~h}$, both with and without an electron acceptor. The TTS apparatus was isolated by adapting a method described by Kubori et al. (1998). The relative amounts of EscC and EscF were measured by immunoblotting with antiEscC and anti-EscF antibodies, respectively. The relative amount of EscF in the TTS apparatus was determined by normalization to the amount of EscC, and it is represented as the fold increase over the amount of the EscC-EscF complex in EHEC grown without an electron acceptor. $\Delta e s c D$, mutant lacking functional TTSS. respiratory systems, such as the TMAO and nitrate respiratory systems.

\section{DISCUSSION}

We demonstrated that the release of type III secreted proteins into the culture supernatant of EHEC grown under anaerobic conditions was much lower than that of EHEC cultured under aerobic conditions. The stimulation of anaerobic growth by adding a specific electron acceptor simultaneously stimulated the secretion of translocators and effectors, represented by EspB and Tir, respectively. The effect of the electron acceptor on EHEC growth appeared to be restricted to the transition phase of growth; the growth rate of EHEC grown with and without an electron acceptor was the same in the exponential phase, while the addition of an electron acceptor to the medium increased the growth rate in the late exponential to early stationary phase. Indeed, the growth rate in the exponential phase for a mutant lacking the gene encoding either TMAO or nitrate reductase was not altered from that of the WT, even in medium containing either TMAO or nitrate, in contrast to the loss of growth stimulation in these mutants at the transition stage (H. Ando \& T. Tobe, unpublished results). The enhancement of TTS at the transition phase was dependent on the activity of the respiratory system specific for the available electron acceptor, given that a mutant lacking either TMAO or nitrate reductase was not able to enhance the secretion of EspB by either TMAO or nitrate, respectively. Furthermore, the inhibition of respiratory activity by amytal abolished the effect of TMAO and nitrate to enhance TTS. The expression of LEE genes, and the release of type III secreted proteins, are optimal in the late exponential phase. Therefore, the activity of the respiratory systems in the transition stage of growth may be critical for the production of functional TTS apparatus.

Although EHEC utilizes all four of the terminal electron acceptors we examined, the effect on the stimulation of TTS was different for each acceptor. Even though DMSO and fumarate could stimulate EHEC growth as much as TMAO and nitrate, the amount of secreted proteins elicited by either DMSO or fumarate was much lower than that elicited by either TMAO or nitrate. These results indicate that stimulation of growth by activating anaerobic respiration is not the reason for TTS activation. Indeed, the increase in ATP levels was not correlated with TTS activity, and the electron acceptor could activate TTS after blocking protein synthesis. It is likely that activation of respiratory systems affects the function of TTSS-associated proteins independently of growth stimulation. We found that EHEC grown without an electron acceptor produced TTS apparatus lacking the EspA filament and EscF needle components at a higher frequency than EHEC grown with either TMAO or nitrate. Since EscC was isolated with TTS apparatus that was partially purified from the membrane of EHEC grown without an electron acceptor, it is unlikely that the translocation of TTSS basal body components to the bacterial 
membrane was impaired when EHEC was grown without an electron acceptor. In S. typhimurium, two forms of TTS apparatus have been found: one is a mature complex with a needle structure, and the other is thought to be a premature form of the complex, lacking the needle structure (Marlovits et al., 2004). Furthermore, assembly of the basal body, which contains outer rings and inner rings, is dependent on Sec machinery, while the next step to form the needle extension is dependent on the components of TTS apparatus (He et al., 2004). Therefore, it is plausible that stimulation of the respiratory system by an electron acceptor enhances the maturation step of TTS apparatus formation, which is dependent on the components of TTS apparatus.

The analysis with mutations in genes encoding quinone synthesis indicates that quinones are necessary for the enhancement of TTS by TMAO and nitrate. Quinones have been shown to modulate the activity of membrane proteins. The oxidized forms of quinones inhibit the kinase activity of ArcB during aerobic growth through the oxidation of two cytosolic redox-active cysteine residues (Georgellis et al., 2001; Malpica et al., 2004). We think that it is likely that quinones play an important role in the formation of the mature TTS apparatus in EHEC by affecting the activity of a component. We propose that oxidized quinones promote conformational changes in a component of TTS apparatus that induces the recruitment of the needle structure to form the mature TTS apparatus. DsbA and DsbB, whose oxidization is dependent on quinones, are known to be required for TTSS activity in Shigella flexneri, Salmonella typhimurium and Yersinia pestis (Watarai et al., 1995; Miki et al., 2004; Jackson \& Plano, 1999). In Y. pestis and S. typhimurium, DsbA is necessary for disulfide bond formation in YscC and SscC, respectively, which are outer-ring components of the TTS basal body. Since translocation of EscC, a homologue of $\mathrm{YscC}$ and $\mathrm{SscC}$, into the macromolecule was observed in EHEC grown without an electron acceptor, it is unlikely that TTS apparatus formation is activated through the activity of DsbA and DsbB. Furthermore, amounts of the oxidized forms of DsbA and DsbB in EHEC were not changed by growth with either TMAO or nitrate, as compared with those with either DMSO or fumarate (H. Ando \& T. Tobe, unpublished results), suggesting that the maturation of TTS apparatus is achieved through a mechanism independent of DsbA and DsbB activity. Since a respiratory system is essential for bacterial growth, it is difficult to distinguish the effect of a deficiency in a respiratory system on TTS function from its effect on protein synthesis or the expression of a functional TTS apparatus in vivo. The identification of a regulatory component that promotes maturation of TTS apparatus would be helpful for the further elucidation of these mechanisms.

\section{ACKNOWLEDGEMENTS}

We thank Akio Abe (Kitasato University) for the antiserum against EscC, Tomoko Kubori and Hiroki Nagai (Osaka University) for technical advice, and Tetsuya Hayashi (University of Miyazaki) for helpful discussion. This work was supported by Grants-in-Aid for Scientific Research on Priority Areas (C) from The Ministry of Education, Culture, Sports, Science, and Technology, Japan.

\section{REFERENCES}

Ansaldi, M., Bordi, C., Lepelletier, M. \& Mejean, V. (1999). TorC apocytochrome negatively autoregulates the trimethylamine $\mathrm{N}$-oxide (TMAO) reductase operon in Escherichia coli. Mol Microbiol 33, 284-295.

Beltrametti, F., Kresse, A. U. \& Guzman, C. A. (1999). Transcriptional regulation of the esp genes of enterohemorrhagic Escherichia coli. J Bacteriol 181, 3409-3418.

Blasco, F., lobbi, C., Ratouchniak, J., Bonnefoy, V. \& Chippaux, M. (1990). Nitrate reductases of Escherichia coli: sequence of the second nitrate reductase and comparison with that encoded by the narGHJI operon. Mol Gen Genet 222, 104-111.

Darwin, A. J. \& Stewart, V. (1995). Nitrate and nitrite regulation of the Fnr-dependent aeg- 46.5 promoter of Escherichia coli K-12 is mediated by competition between homologous response regulators (NarL and NarP) for a common DNA-binding site. J Mol Biol 251, 15-29.

Darwin, A. J., Li, J. \& Stewart, V. (1996). Analysis of nitrate regulatory protein NarL-binding sites in the $f d n G$ and narG operon control regions of Escherichia coli K-12. Mol Microbiol 20, 621-632.

Darwin, A. J., Ziegelhoffer, E. C., Kiley, P. J. \& Stewart, V. (1998). Fnr, NarP, and NarL regulation of Escherichia coli K-12 napF (periplasmic nitrate reductase) operon transcription in vitro. J Bacteriol 180, 4192-4198.

Datsenko, K. A. \& Wanner, B. L. (2000). One-step inactivation of chromosomal genes in Escherichia coli K-12 using PCR products. Proc Natl Acad Sci U S A 97, 6640-6645.

Deng, W., Puente, J. L., Gruenheid, S., Li, Y., Vallance, B. A., Vazquez, A., Barba, J., Ibarra, J. A., O'Donnell, P. \& other authors (2004). Dissecting virulence: systematic and functional analyses of a pathogenicity island. Proc Natl Acad Sci U S A 101, 3597-3602.

Eichelberg, K., Ginocchio, C. C. \& Galan, J. E. (1994). Molecular and functional characterization of the Salmonella typhimurium invasion genes invB and invC: homology of InvC to the F0F1 ATPase family of proteins. J Bacteriol 176, 4501-4510.

Frankel, G., Phillips, A. D., Rosenshine, I., Dougan, G., Kaper, J. B. \& Knutton, S. (1998). Enteropathogenic and enterohaemorrhagic Escherichia coli: more subversive elements. Mol Microbiol 30, 911-921.

Galan, J. E. \& Collmer, A. (1999). Type III secretion machines: bacterial devices for protein delivery into host cells. Science 284, 1322-1328.

Garmendia, J., Frankel, G. \& Crepin, V. F. (2005). Enteropathogenic and enterohemorrhagic Escherichia coli infections: translocation, translocation, translocation. Infect Immun 73, 2573-2585.

Gauthier, A., Puente, J. L. \& Finlay, B. B. (2003). Secretin of the enteropathogenic Escherichia coli type III secretion system requires components of the type III apparatus for assembly and localization. Infect Immun 71, 3310-3319.

Georgellis, D., Kwon, O. \& Lin, E. C. (2001). Quinones as the redox signal for the arc two-component system of bacteria. Science 292, 2314-2316.

Hartland, E. L., Daniell, S. J., Delahay, R. M., Neves, B. C., Wallis, T., Shaw, R. K., Hale, C., Knutton, S. \& Frankel, G. (2000). The type III protein translocation system of enteropathogenic Escherichia coli involves EspA-EspB protein interactions. Mol Microbiol 35, 1483-1492. 
Hayashi, T., Makino, K., Ohnishi, M., Kurokawa, K., Ishii, K., Yokoyama, K., Han, C.-G., Ohtsubo, E., Nakayama, K., Murata, T. \& other authors (2001). Complete genome sequence of enterohemorrhagic Escherichia coli $\mathrm{O} 157: \mathrm{H} 7$ and genomic comparison with laboratory strain K-12. DNA Res 8, 11-22.

He, S. Y., Nomura, K. \& Whittam, T. S. (2004). Type III protein secretion mechanism in mammalian and plant pathogens. Biochim Biophys Acta 1694, 181-206.

Hueck, C. J. (1998). Type III protein secretion systems in bacterial pathogens of animals and plants. Microbiol Mol Biol Rev 62, 379-433.

Ide, T., Laarmann, S., Greune, L., Schillers, H., Oberleithner, H. \& Schmidt, M. A. (2001). Characterization of translocation pores inserted into plasma membranes by type III-secreted Esp proteins of enteropathogenic Escherichia coli. Cell Microbiol 3, 669-679.

Jackson, M. W. \& Plano, G. V. (1999). DsbA is required for stable expression of outer membrane protein YscC and for efficient Yop secretion in Yersinia pestis. J Bacteriol 181, 5126-5130.

Kenny, B., Abe, A., Stein, M. \& Finlay, B. B. (1997). Enteropathogenic Escherichia coli protein secretion is induced in response to conditions similar to those in the gastrointestinal tract. Infect Immun 65, 2606-2612.

Knutton, S., Rosenshine, I., Pallen, M. J., Nisan, I., Neves, B. C., Bain, C., Wolff, C., Dougan, G. \& Frankel, G. (1998). A novel EspA-associated surface organelle of enteropathogenic Escherichia coli involved in protein translocation into epithelial cells. Embo J 17, 2166-2176.

Kubori, T., Matsushima, Y., Nakamura, D., Uralil, J., Lara-Tejero, M., Sukhan, A., Galan, J. E. \& Aizawa, S. I. (1998). Supramolecular structure of the Salmonella typhimurium type III protein secretion system. Science 280, 602-605.

Laemmli, U. K. (1970). Cleavage of structural proteins during the assembly of the head of bacteriophage T4. Nature 227, 680-685.

Lee, P. T., Hsu, A. Y., Ha, H. T. \& Clarke, C. F. (1997). A Cmethyltransferase involved in both ubiquinone and menaquinone biosynthesis: isolation and identification of the Escherichia coli ubiE gene. J Bacteriol 179, 1748-1754.

Malpica, R., Franco, B., Rodriguez, C., Kwon, O. \& Georgellis, D. (2004). Identification of a quinone-sensitive redox switch in the ArcB sensor kinase. Proc Natl Acad Sci U S A 101, 13318-13323.

Marlovits, T. C., Kubori, T., Sukhan, A., Thomas, D. R., Galan, J. E. \& Unger, V. M. (2004). Structural insights into the assembly of the type III secretion needle complex. Science 306, 1040-1042.

Mejean, V., lobbi-Nivol, C., Lepelletier, M., Giordano, G., Chippaux, M. \& Pascal, M. C. (1994). TMAO anaerobic respiration in Escherichia coli: involvement of the tor operon. Mol Microbiol 11, 1169-1179.

Miki, T., Okada, N. \& Danbara, H. (2004). Two periplasmic disulfide oxidoreductases, DsbA and SrgA, target outer membrane protein SpiA, a component of the Salmonella pathogenicity island 2 type III secretion system. J Biol Chem 279, 34631-34642.

Moon, H. W., Whipp, S. C., Argenzio, R. A., Levine, M. M. \& Giannella, R. A. (1983). Attaching and effacing activities of rabbit and human enteropathogenic Escherichia coli in pig and rabbit intestines. Infect Immun 41, 1340-1351.

Nataro, J. P. \& Kaper, J. B. (1998). Diarrheagenic Escherichia coli. Clin Microbiol Rev 11, 142-201.

O'Connell, C. B., Creasey, E. A., Knutton, S., Elliott, S., Crowther, L. J., Luo, W., Albert, M. J., Kaper, J. B., Frankel, G. \& Donnenberg, M. S. (2004). SepL, a protein required for enteropathogenic Escherichia coli type III translocation, interacts with secretion component SepD. Mol Microbiol 52, 1613-1625.

Ogura, Y., Kurokawa, K., Ooka, T., Tashiro, K., Tobe, T., Ohnishi, M., Nakayama, K., Morimoto, T., Terajima, J. \& other authors (2006).
Complexity of the genomic diversity in enterohemorrhagic Escherichia coli $\mathrm{O} 157$ revealed by the combinational use of the O157 Sakai oligoDNA microarray and the whole genome PCR scanning. DNA Res 13, 3-14.

Ohnishi, M., Terajima, J., Kurokawa, K., Nakayama, K., Murata, T., Tamura, K., Ogura, Y., Watanabe, H. \& Hayashi, T. (2002). Genomic diversity of enterohemorrhagic Escherichia coli O157 revealed by whole genome PCR scanning. Proc Natl Acad Sci U S A 99, 17043-17048.

Pommier, J., Mejean, V., Giordano, G. \& Iobbi-Nivol, C. (1998). TorD, a cytoplasmic chaperone that interacts with the unfolded trimethylamine $\mathrm{N}$-oxide reductase enzyme (TorA) in Escherichia coli. J Biol Chem 273, 16615-16620.

Schagger, H. \& von Jagow, G. (1987). Tricine-sodium dodecyl sulfate-polyacrylamide gel electrophoresis for the separation of proteins in the range from 1 to $100 \mathrm{kDa}$. Anal Biochem 166, 368-379.

Schauer, D. B. \& Falkow, S. (1993). Attaching and effacing locus of a Citrobacter freundii biotype that causes transmissible murine colonic hyperplasia. Infect Immun 61, 2486-2492.

Sekiya, K., Ohishi, M., Ogino, T., Tamano, K., Sasakawa, C. \& Abe, A. (2001). Supermolecular structure of the enteropathogenic Escherichia coli type III secretion system and its direct interaction with the EspAsheath-like structure. Proc Natl Acad Sci U S A 98, 11638-11643.

Shalel-Levanon, S., San, K. Y. \& Bennett, G. N. (2005). Effect of ArcA and FNR on the expression of genes related to the oxygen regulation and the glycolysis pathway in Escherichia coli under microaerobic growth conditions. Biotechnol Bioeng 92, 147-159.

Stevenson, K. S. D., Meganathan, R. \& Hudspeth, M. E. (1998). Menaquinone (vitamin $\mathrm{K}_{2}$ ) biosynthesis: localization and characterization of the menA gene from Escherichia coli. J Bacteriol 180, 2782-2787.

Stewart, V. (2003). Biochemical Society Special Lecture. Nitrate- and nitrite-responsive sensors NarX and NarQ of proteobacteria. Biochem Soc Trans 31, 1-10.

Tatsuno, I., Kimura, H., Okutani, A., Kanamura, K., Abe, H., Nagai, S., Makino, K., Shinagawa, H., Yoshida, M. \& other authors (2000). Isolation and characterization of mini-Tn $5 \mathrm{Km} 2$ insertion mutants of enterohemorrhagic Escherichia coli $\mathrm{O} 157: \mathrm{H} 7$ deficient in adherence to Caco-2 cells. Infect Immun 68, 5943-5952.

Tzipori, S., Gunzer, F., Donnenberg, M. S., de Montigny, L., Kaper, J. B. \& Donohue-Rolfe, A. (1995). The role of the eaeA gene in diarrhea and neurological complications in a gnotobiotic piglet model of enterohemorrhagic Escherichia coli infection. Infect Immun 63, 3621-3627.

Walker, M. S. \& DeMoss, J. A. (1994). NarL-phosphate must bind to multiple upstream sites to activate transcription from the narG promoter of Escherichia coli. Mol Microbiol 14, 633-641.

Watarai, M., Tobe, T., Yoshikawa, M. \& Sasakawa, C. (1995). Disulfide oxidoreductase activity of Shigella flexneri is required for release of Ipa proteins and invasion of epithelial cells. Proc Natl Acad Sci U S A 92, 4927-4931.

Wilson, R. K., Shaw, R. K., Daniell, S., Knutton, S. \& Frankel, G. (2001). Role of EscF, a putative needle complex protein, in the type III protein translocation system of enteropathogenic Escherichia coli. Cell Microbiol 3, 753-762.

Wissenbach, U., Ternes, D. \& Unden, G. (1992). An Escherichia coli mutant containing only demethylmenaquinone, but no menaquinone: effects on fumarate, dimethylsulfoxide, trimethylamine $\mathrm{N}$ oxide and nitrate respiration. Arch Microbiol 158, 68-73.

Woestyn, S., Allaoui, A., Wattiau, P. \& Cornelis, G. R. (1994). YscN, the putative energizer of the Yersinia Yop secretion machinery. J Bacteriol 176, 1561-1569.

Edited by: B. Kenny 